\title{
FRACCIONAMIENTO QUÍMICO DE LA MATERIA ORGÁNICA EN TRES SUELOS DE LA COLONIA TOVAR - VENEZUELA
}

\section{CHEMICAL FRACTIONATION OF ORGANIC MATTER THREE SOIL IN COLONIA TOVAR - VENEZUELA}

\author{
Eliecer M. Cabrales ${ }^{1}$, Jairo Ferrer², Rosa M. Hernández ${ }^{3}$ \\ Recibido para publicación: Abril 28 de 2011 - Aceptado para publicación: Diciembre 22 de 2011
}

\begin{abstract}
RESUMEN
Esta investigación se realizó en suelos de pequeños productores de la Colonia Tovar - Venezuela, a una altura aproximada de 2000 m.s.n.m, clima templado de montaña. El muestreo se hizo en los primeros $20 \mathrm{~cm}$ de profundidad en transectas de $50 \mathrm{~m}$ en tres localidades con distinto uso: bosque, cultivos de fresa y cultivo de durazno. El fraccionamiento químico de la materia orgánica: ácidos fúlvicos (CAF), ácidos húmicos (CAH) y sustancias no húmicas (CSNH), se hizo por el método Schinitzer y Schuppli y el carbono orgánico total (COT) y carbono orgánico de cada fracción por el método de oxidación en húmedo de Walkley y Black modificado por Heanes. Con base en estos resultados se determinaron los índices de humificación. Se utilizó un diseño completamente aleatarizado con cinco repeticiones, para lo cual se utilizó el paquete estadístico Statistic 8,0. Se obtuvieron diferencias altamente significativas en todos los parámetros evaluados, siendo el bosque quien se mantiene por encima en todos los casos: 32,3; 9,7; 10,9; 53,0 y 61,2 g. $\mathrm{kg}^{-1}$ de carbono para CAH, CAF, CSNH, Cext y COT respectivamente. De acuerdo con los índices, se pudo notar que el bosque posee una materia orgánica más estable, compleja y con un mayor grado de humificación (79\%). Se concluyo que los cultivos disminuyen el contenido de carbono orgánico en todas las fracciones de la materia orgánica de estos suelos, al igual que su grado de polimerización.
\end{abstract}

Palabras clave: carbono orgánico, ácidos húmicos, ácidos fúlvicos, sustancias no húmicas, índices de humificación.

\begin{abstract}
This research was conducted on soils of small farmers in the Colonia Tovar - Venezuela, with an approximate height of $2000 \mathrm{~m}$ over the sea level, characterized by its template mountain weather. The sampling was done

\footnotetext{
1M.Sc., Docente Área de suelos, Universidad de Córdoba, Montería - Colombia, telefax 4-7860255 ecabralesh@yahoo.es ${ }^{2}$ M.Sc., Docente área de Edafología, Universidad Experimental Rómulo Gallego, San Juan de los Morros - Venezuela jairofe@yahoo.com.

${ }^{3}$ Ph.D., Docente área Edafología, Universidad Experimental Simón Rodríguez, Caracas - Venezuela rosa.hernandez@ unesr.edu.ve
} 
in the first $20 \mathrm{~cm}$ depth along $50 \mathrm{~m}$ transects, in three locations with different uses: forest, growing crops of strawberry and peach. All samples were processed for organic matter chemical fractionation. Fulvic acids, humic acids and no-humic substances by the Schnitzer and Schuppli method; total organic carbon (TOC) and organic carbon in each fraction by the Walkley and Black wet oxidation method modified for Heanes. Based on these results we determined the indices of humification. Randomized complete block with five repetitions design was used, the information were analyzed with Statistic 8,0 statistical package. In all parameters evaluated, highly significant differences were obtained. The forest remains higher in all cases: 32,$3 ; 9,7 ; 10,9$; 53,0 and $61,2 \mathrm{~g} \cdot \mathrm{kg}^{-1}$ of carbon for CAH, CAF, CSNH, Cext y COT respectively. According to the indexes, it was noted that the forest has a more stable and complex organic matter, and has a higher degree of humification $(79 \%)$. Was concluded that the crop decrease the organic carbon content in all fractions of organic matter in these soils, as well as its degree of polymerization.

Key words: organic carbon, humic acids, fulvic acids, no-humic substances, humification index.

\section{INTRODUCCIÓN}

La dinámica de la materia orgánica del suelo (MOS) es un tema de mucho interés en la sostenibilidad de los mismos, toda vez que en ella se alberga la biota edáfica responsable de dicha actividad, que es gobernada hasta cierto punto por las propiedades físicas y químicas del suelo. La MOS está comprendida por una mezcla compleja y heterogénea de sustancias o residuos orgánicos de diversa naturaleza, sometidos a una continua transformación a través de factores biológicos, químicos y físicos (Sánchez et al. 2005), toda esta mezcla compleja representa aproximadamente el 10$15 \%$ de la reserva total de materia orgánica del suelo (Zagal y Córdova 2005).

La materia orgánica humificada incrementa la estabilidad de los agregados del suelo y disminuye la densidad aparente del mismo; en el mismo sentido, los agentes de unión de tipo persistente y responsables de la microagregación, aumentan muy poco el tamaño de éstos agregados, toda vez que actúan principalmente a escala del tamaño de las partículas de minerales de arcilla. En este sentido, la presencia de ácidos húmicos
(CAH) y ácidos fúlvicos (CAF) en suelos de textura liviana podrían ayudar a estabilizar su estructura.

Los ácidos húmicos caracterizados por su alto peso molecular y alta capacidad de intercambio de cationes (CIC), son los más importantes en el proceso de estabilización de los agregados, contrario a lo señalado para los ácidos fúlvicos. Sin embargo, otros autores consideran que las sustancias húmicas de bajo peso molecular ejercen mayor acción en la agregación del suelo, toda vez que poseen mayor cantidad de grupos funcionales oxigenados libres como los carboxilos, oxhidrilos fenólicos y carbonilos (-COOH, -OH y -COO) (López et al. 2006).

La MOS varía desde los residuos de cultivos y animales recientemente incorporados hasta la compleja estructura del humus alcanzada luego de siglos de transformación (Galantini y Suñer 2008). Sin embargo, la materia orgánica activa del suelo que participa en la agregación y propiedades químicas del suelo, consiste principalmente de material húmico, de carácter aromático, recalcitrante, y algunos biopolímeros como los polisacáridos. Se ha comprobado que la estabilidad de 
los microagregados se incrementa ante la presencia de cationes polivalentes en el suelo (Ruiz et al. 2000).

Los suelos agrícolas se caracterizan por contener menor cantidad de materia orgánica que los suelos de bosque, y en la medida que son mas laboreados, menor es su contenido. Se ha planteado que en suelos agrícolas de zonas templadas, un $1 \%$ de carbono podría representar el umbral, por debajo del cual, el funcionamiento del sistema suelo/ cultivo podría no ser exitoso, aun cuando se le suministren los fertilizantes minerales adecuados (Romanyà et al. 2007).

Con base en lo anterior, el presente estudio tuvo como finalidad determinar los cambios que se producen en la humificación de las fracciones más estables de la MOS, como las sustancias húmicas, en suelos de bosque nublado, cuando son cultivados con fresa y durazno.

\section{MATERIALES Y MÉTODOS}

La investigación se realizó en la serranía del Litoral Central de la cordillera de la Costa, estado Aragua-Venezuela, en ecosistemas de clima templado de montaña y agroecosistemas de cultivos de fresas y duraznos de pequeños productores de La Colonia Tovar. La zona se encuentra ubicada a una altura aproximada de 2000 m.s.n.m., y está caracterizada por sus amplitudes térmicas diarias de unos de $10^{\circ} \mathrm{C}$, con promedio de $16,8^{\circ} \mathrm{C}$ (Sánchez 1981). Las pendientes del relieve son muy escarpadas encontrándose valores por encima del 55\%. Los suelos de la zona se encuentran clasificados (Mogollón y Comerma (1994) y Herrera y Cuevas (2003)) dentro del Gran Grupo de los Humitropepts, caracterizados por una incipiente evolución pedogenética, presentando además acumulaciones de materia orgánica en la superficie. La región se caracteriza por ser una zona agrícola y turística por excelencia.

En relación al manejo de los cultivos, la fresa se cosecha dos veces por semana y el suelo se mantiene recubierto con plástico para mantener el terreno húmedo e impedir la emergencia de malezas. Para este cultivo, se usan abonos orgánicos como cascarilla de arroz y estiércol de conejo y aves, en algunos casos, con abonos inorgánicos de tipo fórmulas completas de N-P-K. El manejo de plagas y enfermedades se hace mediante el uso de pesticidas químicos. Mientras que el cultivo de durazno, es poco abonado y su ciclo puede durar hasta 5 o 6 años, al igual que la fresa, el control de plagas y enfermedades se hace mediante prácticas químicas.

El muestreo de suelos se hizo en los primeros $20 \mathrm{~cm}$ de profundidad en tres localidades: zona de bosque, zona de cultivos de fresa y durazno. El bosque no había sido intervenido antrópicamente y estaba adyacente a las zonas de los cultivos de fresa y durazno, los cuales tienen más de cinco años de explotación. La pendiente en los lotes de muestreo fue similar y osciló entre 25 y 30\%. En cada zona y en un área de aproximadamente $1000 \mathrm{~m}^{2}$, se levantaron cinco transectas perpendiculares a la pendiente y se tomó una muestra compuesta a partir de 10 submuestras tomadas a lo largo de cada transecta, cuya longitud fue de unos $40 \mathrm{~m}$ aproximadamente. Las muestras se homogeneizaron, se secaron a la sombra, se 
pasaron por el tamiz de $2 \mathrm{~mm}$. Se determinó la textura por el método de Boyoucos, $\mathrm{pH}$ en relación suelo agua 1:1, fósforo por Holsen, calcio y potasio por extracción con acetato de amonio $1 \mathrm{M}$ y neutro (Anderson e Ingram, 1993). El fraccionamiento químico de estas muestras (ácidos fúlvicos (CAF), ácidos húmicos $(\mathrm{CAH})$ y sustancias no húmicas (CSNH) se hizo por el método Schinitzer y Schuppli (1989), el carbono orgánico total y el carbono orgánico de cada fracción se hizo por el método de oxidación en húmedo de Walkley y Black modificado (Heanes 1984). Con base en los resultados obtenidos, se calcularon los parámetros de humificación propuestos por Acosta et al. (2004):

Índice de humificación

$(\mathrm{IH}): \mathrm{IH}=\mathrm{CSNH} /(\mathrm{CAH}+\mathrm{CAF})$

Grado de humificación

$(\mathrm{GH}): \mathrm{GH}=((\mathrm{CAH}+\mathrm{CAF}) / \mathrm{CET}) * 100$

Tasa de humificación

$(\mathrm{TH}): \mathrm{TH}=((\mathrm{CAH}+\mathrm{CAF}) / \mathrm{COT}) * 100$

Relación CAF/CAH: CAF/CAH

Grado de polimerización (GP): GP $=\mathrm{CAH} / \mathrm{CAF}$

Relación de humificación

$(\mathrm{RH}): \mathrm{RH}=\mathrm{Cext} / \mathrm{COT}^{*} 100$

Donde: $\mathrm{CAH}=$ carbono de ácidos húmicos, $\mathrm{CAF}=$ carbono de ácidos fúlvicos, $\mathrm{CSNH}=$ carbono de sustancias no humitas, Cext
$=$ carbono extraíble total, COT = carbono orgánico total.

El procesamiento estadístico se realizó con el paquete Statistic 8,0, de forma completamente aleatorizada con cinco repeticiones por uso. Se ejecutó la prueba de normalidad de ShapiroWills modificado y para el análisis de varianza se utilizó la comparación de medias por el test de rango múltiple DLS (diferencia mínima significativa), con un nivel de significancia del $95 \%$ ( $\leq \leq 0,05)$, mientras que las correlaciones se hicieron por el método de Pearson.

\section{RESULTADOS Y DISCUSIÓN}

El suelo bajo vegetación natural de Bosque presenta texturas franca a franca arenosa, $\mathrm{pH}$ fuertemente ácido y bajos valores de los atributos químicos (Tabla 1), ello concuerda con lo reportado en la literatura para la zona (Jaimes 1988; Avilán et al. 1975). En contraste en los suelos intervenidos con fresa y durazno, el $\mathrm{pH}$ va de moderada a ligeramente ácido, el contenido de calcio, potasio y fósforo es alto en los suelos cultivados con fresa y durazno, mientras que para el bosque suele ser de contenidos medios a bajo (Tabla 1). El aumento de los niveles de nutrimentos se debe principalmente a que en estos sistemas de producción son adicionados abonos orgánicos

Tabla 1. Características Físico químicas de tres suelos de la Colonia Tovar - Venezuela según el uso.

\begin{tabular}{|c|c|c|c|c|c|c|c|c|c|}
\hline \multirow{2}{*}{ USO } & \multirow{2}{*}{ Textura $^{1}$} & \multirow{2}{*}{$\mathrm{pH}^{2}$} & \multirow{2}{*}{$\begin{array}{c}\mathrm{CE}^{3} \\
\mathrm{dS}^{*} \mathrm{~m}^{-1}\end{array}$} & \multicolumn{2}{|c|}{ Calcio } & \multicolumn{2}{|c|}{ Potasio } & \multicolumn{2}{|c|}{ Fósforo } \\
\hline & & & & $\mathrm{mg}^{*} \mathrm{~kg}^{-1}$ & CV $(\%)^{4}$ & $\mathrm{mg}^{*} \mathbf{k g}^{-1}$ & CV $(\%)^{4}$ & $\mathrm{mg}^{*} \mathrm{~kg}^{-1}$ & CV $(\%)^{4}$ \\
\hline Bosque & FA & $(4,2-4,5)$ & 0,06 & 170 & 11,6 & 26,8 & 12,5 & 9 & 31,5 \\
\hline Fresa & $\mathrm{F}$ & $(5,5-5,8)$ & 0,41 & 1444 & 4 & 116,8 & 23,4 & 160 & 2,6 \\
\hline Durazno & FAa & $(5,2-6,1)$ & 0,15 & 1504 & 23,3 & 76 & 31,8 & 156 & 5,4 \\
\hline
\end{tabular}

${ }^{1}$ Textura $=$ Clasificación USDA; ${ }^{2} \mathrm{pH}=$ Agua $1: 1 ;{ }^{3} \mathrm{CE}=$ Conductividad Eléctrica; ${ }^{4} \mathrm{CV}=$ Coeficiente de Variación; $\mathrm{dS} * \mathrm{~m}^{-1}=$ decisiemens $\mathrm{x}$ metro; $\mathrm{mg}^{*} \mathrm{~kg}^{-1}=$ miligramo $\mathrm{x}$ kilogramo de suelo. 
en cantidades elevadas sin tener presentes los análisis de suelos.

Los resultados del fraccionamiento químico de la materia orgánica de los suelos en estos sistemas de explotación señalan que hay diferencias altamente significativas, es decir, que el manejo de suelos influye grandemente en las fracciones químicas de la materia orgánica de los mismos, siendo el bosque quien se mantiene por encima en todos los casos (Tabla 2). En los suelos sometidos a manejo agrícola las fracciones químicas de la materia orgánica del suelo bajan en cantidad, aunque, son similares independientemente del rubro, ya que ambos (fresa y durazno) tienen un manejo intensivo. Esta tendencia concuerda con lo reportado por Piccolo et al. (1998) cuando comparó un suelo virgen con distintos tipos de manejo de suelos, en un bosque subtropical húmedo con más de 50 años de edad.

El mayor contendido de carbono orgánico se encontró en los ácidos húmicos $(\mathrm{AH})$ presentes en el bosque, con concentraciones de 32,3 g. $\mathrm{kg}^{-1}$ de carbono, con diferencias altamente significativas respecto a los otros dos usos y la más baja concentración se encontró en el cultivo de fresa con 20,19 g. $\mathrm{kg}^{-1}$ de carbono; esta ultima sin diferencia significativa con respecto al cultivo de durazno. Estos resultados indican que las prácticas de manejo del suelo influyen notablemente en el contenido de $\mathrm{AH}$ del suelo. Estos contenidos están muy por encima de los 11,27 g. $\mathrm{kg}^{-1}$ de carbono en $\mathrm{AH}$ que reporta Machado et al. (2008) en suelos brasileros dedicados a diversos cultivos durante varios años, también están por encima de los 0,9 - 4,0 g. $\mathrm{kg}^{-1}$ de carbono que reporta Armado et al (2009) en suelos cacaoteros de Venezuela. Igualmente valores más bajos de $\mathrm{AH}$ fueron reportados por Pulido-Moncada et al. (2010), en suelos inceptisoles y ultisoles bajo bosques $\left(\leq 8,65 \mathrm{~g} \cdot \mathrm{kg}^{-1}\right.$ de carbono) y bajo uso de cítricas $\left(\leq 5,06\right.$ g. $\left.\mathrm{kg}^{-1}\right)$ y por Figuera et al. (2005) en suelos vertisoles, entisoles y ultisoles $(\leq 2,47$ g. $\mathrm{kg}^{-1}$ ) en sabanas bajo pastoreo extensivo por 30 años, en los Ilanos Venezolanos.

El carbono orgánico de los ácidos fúlvicos (AF) también fue mayor en los suelos de bosques, indicando también que las prácticas de manejo disminuye el contenido de estos ácidos en estos suelos de bosque nublado, lo que concuerda con Piccolo et al. (1998) en cuanto al efecto de uso de la tierra en los contenidos de ácidos

Tabla 2. Fraccionamiento químico de la materia orgánica de tres suelos de la Colonia Tovar - Venezuela según el uso.

\begin{tabular}{|c|c|c|c|c|c|}
\hline \multirow{2}{*}{ Uso } & $\mathrm{CAH}^{1}$ & $\mathrm{CAF}^{2}$ & $\mathrm{CSNH}^{3}$ & Cext $^{4}$ & $\mathrm{COT}^{5}$ \\
\hline & \multicolumn{5}{|c|}{ g.kg-1 de carbono } \\
\hline Bosque & $32,36 a^{*}$ & $9,76 \mathrm{a}$ & $10,95 \mathrm{a}$ & $53,08 \mathrm{a}$ & $61,23 \mathrm{a}$ \\
\hline Fresa & $20,19 \mathrm{~b}$ & $7,66 \mathrm{~b}$ & $9,23 \mathrm{~b}$ & $37,08 \mathrm{~b}$ & $38,92 \mathrm{~b}$ \\
\hline Durazno & $21,23 \mathrm{~b}$ & $7,26 \mathrm{~b}$ & $9,13 \mathrm{~b}$ & $37,62 \mathrm{~b}$ & $39,20 \mathrm{~b}$ \\
\hline $\mathrm{CV}(\%)^{6}$ & 20,91 & 12,02 & 9,07 & 12,46 & 10,11 \\
\hline
\end{tabular}

${ }^{1} \mathrm{CAH}=$ Carbono orgánico de ácidos húmicos; ${ }^{2} \mathrm{CAF}=$ Carbono orgánico de ácidos fúlvicos; ${ }^{3} \mathrm{CSNH}=\mathrm{Carbono}$ orgánico de sustancias no húmicas; ${ }^{4} \mathrm{Cext}=$ Carbono orgánico extraíble total; ${ }^{5} \mathrm{COT}=$ Carbono orgánico total; ${ }^{6} \mathrm{CV}=\mathrm{Coeficiente}$ de Variación.

*Letras distintas en sentido vertical difieren significativamente $(p<0,05)$ 
fúlvicos en los mismos. El mayor contenido de AF se encontró en el bosque con 9,76 g. $\mathrm{kg}^{-1}$ de carbono y el de menor valor en el cultivo de durazno con 7,26 g. $\mathrm{kg}^{-1}$, sin embargo, entre este último y la fresa, no hubo diferencia significativa. Estos hallazgos son similares a los que reporta Machado et al. (2008) en suelos agrícolas brasileros, los cuales oscilan alrededor de 7,95 g. $\mathrm{kg}^{-1}$. Sin embargo, en Venezuela se han reportado valores $\leq 2,8 \mathrm{~g} \cdot \mathrm{kg}^{-1}$ de carbono para cultivo de Cacao (Armado et al. 2009), $\leq 1,09$ g. $\mathrm{kg}^{-1}$ en sabanas naturales pastoreadas (Figuera et al. 2005), $\leq 3,25$ g. $\mathrm{kg}^{-1}$ bajo bosque y $\leq 2,62 \mathrm{~g} \cdot \mathrm{kg}^{-1}$ en cítricas (Pulido-Moncada et al. 2010), todos ellos muy inferiores a los obtenidos en la presente investigación.

Igualmente las sustancias no-húmicas $(\mathrm{CSNH})$ mostraron el mismo comportamiento puesto el bosque presentó los valores más altos en esa fracción de MOS en relación a los suelos bajo cultivos. Existe diferencia significativa $(p<0,05)$ entre el bosque y los sistemas de explotación, pero no se produjo un efecto por el tipo de manejo agrícola. Este resultado permite afirmar que en estos suelos, los sistemas agrícolas contribuyen a la disminución no solo de las sustancias húmicas, sino también de las no-húmicas. La mayor concentración de carbono orgánico en estas CSNH se encontró en los suelos del bosque con 10,95 g. $\mathrm{kg}^{-1}$ de carbono mientras que la de menor valor fue la de durazno con 9,13 g. $\mathrm{kg}^{-1}$ de carbono. Estos valores están muy por encima de los 3,9 g. $\mathrm{kg}^{-1}$ de carbono que reporta Armado et al (2009) en suelo cacaoteros de Venezuela, así como de los obtenidos por Figuera et al. (2005) en suelos de los Ilanos Venezolanos bajo pastoreo ( $\leq 5,02$ g. $\mathrm{kg}^{-1}$ de carbono).
Para el carbono orgánico extraíble (Cext), se pudo notar también una mayor concentración de esta fracción en suelos de bosque y una disminución en suelos cultivados en fresa y durazno. Esta diferencia significativa presumiblemente obedece a que el bosque posee mayor contenido de materia orgánica total y que esta ha disminuido en la medida que son intervenidos para el uso en agricultura. El mayor valor se encontró en suelos de bosque con 53,08 g. $\mathrm{kg}^{-1}$ de carbono mientras que el de menor valor fue el suelo de fresa con 37,08 g. $\mathrm{kg}^{-1}$ de carbono. Estos valores están muy por encima de los $11 \mathrm{~g} \cdot \mathrm{kg}^{-1}$ de carbono que reportan en suelos agrícolas Venezolanos (Pulido-Moncada et al. 2010; Armado et al. 2009; Figuera et al. 2005; Lozano et al. 2002). Es esperable que el Cext sea mayor en el bosque porque este ecosistema presentó en sus suelos las mayores fracciones de ácidos húmicos, fúlvicos y sustancias no húmicas.

El carbono orgánico total (COT) de los suelos estudiados es bastante alto cuando se les compara con lo reportado en estudios de suelos agrícolas Venezolanos (Pulido-Moncada et al. 2010; Armado et al. 2009; Figuera et al. 2005; Lozano et al. 2002), siendo el mayor valor el encontrado bajo bosque con 61,23 g. kg-1 de carbono y el de menor valor en durazno, sin diferencias entre los suelos cultivados en fresa y durazno, pero si entre estos dos suelos y el de bosque.

Los resultados contribuyen a confirmar que el uso agrícola del suelo, favorece el detrimento de los ácidos húmicos y fúlvicos, así como también de las sustancias no húmicas en él. De los suelos estudiados el de uso bajo bosque es rico en $\mathrm{MO}$ debido al balance favorable de 
la relación Precipitación/Evapotranspiración en la zona, lo que beneficia el desarrollo de una vegetación densa con aportes continuos de biomasa de hojarasca, la cual conforma un colchón de unos $10 \mathrm{~cm}$ de grosor sobre la superficie del suelo. Este aporte por biomasa de hojarasca se pierde cuando se produce el cambio de uso de la tierra de bosque a cultivo. No obstante, en los suelos intervenidos con fresa y durazno, el contenido relativamente alto de $\mathrm{MO}$ se debe a la aplicación de abonos orgánicos usando estiércol de conejo, práctica típica de estos sistemas de producción en el área. Sin embargo, dada las altas pendientes que caracterizan a estas zonas en particular, cuando los terrenos no son protegidos con algún tipo de cobertura, es posible que parte de este abono pueda perderse por lavado y/o percolado. Igualmente este abono orgánico puede descomponerse relativamente rápido favorecido por la humedad generada por el microclima o el riego. Lo anterior pudiera explicar los mayores valores de C presentes en estos sistemas con respecto a los reportados en otras zonas bajo usos agrícolas del país.

Los índices derivados de las fracciones químicas de la materia orgánica plasmados en el Tabla 3, muestran que el bosque exhibe un menor valor de índice de humificación (IH), esto estaría indicando que posee una mayor proporción de carbono humificado (CAH, CAF) frente al no humificado (CSNH). En todos los casos, el índice de humificación (IH) fue bajo $(<1)$ lo que sugiere que el tipo de MO tiende a ser relativamente estable, en la mayor parte de los casos, los valores que concuerdan con lo reportado por Armado et al. (2009), Acosta et al (2004) y Lozano et al. (2002). Por su parte Figuera et al. (2005) reportan valores mayores a uno en suelos de los Ilanos Venezolanos bajo pastoreo, sin embargo indican que esto se vio afectado por un bajo porcentaje de recuperación de las fracciones.

Los valores anteriores del IH están también relacionados con lo obtenido en el grado de humificación $(\mathrm{GH})$ que fue $>74 \%$, lo cual es similar a lo reportado por Lozano et al. (2002) en entisoles, ultisoles y vertisoles con gramíneas nativas bajo pastoreo de los Ilanos Venezolanos y Pulido-Moncada et al. (2010) en inceptisoles bajo Bosque y Cítricas en el estado Carabobo, Venezuela. Valores > 60\% indican materiales muy humificados (Acosta et al. 2004).

Los valores obtenidos de la tasa de humificación (TH) son similares entre sí, siendo relativamente altos (alrededor del 70\%), por lo que se prevé que esta $\mathrm{MO}$ puede humificarse en un alto grado y con ello perdurar más

Tabla 3. Índices derivados de las fracciones químicas de la materia orgánica de tres suelos de la Colonia Tovar - Venezuela según el uso.

\begin{tabular}{|c|c|c|c|c|c|c|c|}
\hline Uso & $\mathrm{IH}^{1}$ & $\mathrm{GH}^{2}$ & $\mathrm{TH}^{3}$ & $G^{4}$ & $\mathbf{R H}^{5}$ & $\mathrm{CAF}^{6} / \mathrm{CAH}^{7}$ & $\mathrm{CAF} / \mathrm{COT}^{8}$ \\
\hline Bosque & 0,27 & 79,04 & 68,78 & 3,39 & 86,88 & 0,32 & 16,17 \\
\hline Fresa & 0,34 & 74,90 & 71,41 & 2,65 & 95,26 & 0,38 & 19,74 \\
\hline Durazno & 0,32 & 75,67 & 72,59 & 2,92 & 95,98 & 0,34 & 18,52 \\
\hline CV $(\%)^{9}$ & 15,92 & 5,77 & 9,20 & 19,22 & 7,68 & 15,21 & 15,23 \\
\hline
\end{tabular}

${ }^{1} \mathrm{IH}=$ Índice de humificación; ${ }^{2} \mathrm{GH}=$ Grado de humificación; ${ }^{3} \mathrm{TH}=$ Tasa de humificación; ${ }^{4} \mathrm{GP}=$ Grado de polimerización; ${ }^{5} \mathrm{RH}=$ Relación de humificación; ${ }^{6} \mathrm{CAF}=$ Carbono orgánico de ácidos fúlvicos; ${ }^{7} \mathrm{CAH}=$ Carbono orgánico de ácidos húmicos; ${ }^{8} \mathrm{COT}=$ Carbono orgánico total; ${ }^{9} \mathrm{CV}=$ Coeficiente de Variación. 
tiempo en el suelo, toda vez que un material humificado suele tener una descomposición mucho más baja. El mayor valor del grado de polimerización (GP) fue de 3,39 para el Bosque, el cual disminuyó con el cambio de uso de la tierra, siendo 2,92 en Durazno y 2,65 en Fresa. Esto está en concordancia con la relación CAF/CAH cuyos valores son menores a 0,4. Estas dos últimas relaciones mencionadas, nos están indicando por un lado un predominio del $\mathrm{CAH}$ sobre el CAF, y por el otro, que el manejo de fresa y durazno está haciendo disminuir esa predominancia de los Ácidos Húmicos respecto al bosque. En consecuencia, aún cuando el $\mathrm{IH}$ y el GH indican predominio del $\mathrm{C}$ humificado, el GP está indicando que el CAH disminuye en los suelos intervenidos con fresa y durazno en relación con los suelos de bosque.

Cuando se relaciona lo mencionado en el párrafo anterior, con los valores de CAF/COT, se observa que este porcentaje es menor en el suelo bajo bosque (16,17\%), aumentando a 18,52 y $19,74 \%$ cuando el bosque es sustituido por cultivos de durazno y fresa, respectivamente. Esto hace suponer que el cambio de uso de bosque a uso agrícola puede contribuir a la disminución de formación de compuestos con menor grado de polimerización, lo que los hace susceptible a degradarse mas aceleradamente por el manejo (Zalba y Quiroga 1999), a lo que se suma también las pérdidas por erosión dado el alto grado de pendiente de estos suelos, los cuales pueden estar más desprotegidos en caso de los cultivos. Es necesario considerar, en los manejos de fresas y de duraznos, el uso de prácticas que favorezcan no solo la restitución de $\mathrm{MO}$ en los suelos, sino minimizar las pérdidas de las aplicadas mediante prácticas de coberturas.
En el mismo sentido, se hicieron correlaciones entre las variables del fraccionamiento químico, incluido sus índices, las mejores correlaciones se encontraron entre ácidos húmicos $(\mathrm{CAH})$ y carbono orgánico total (COT) con 88\%, mientras que la de los ácidos fúlvicos (CHF) con carbono orgánico total, carbono orgánico extraíble (CEXT) y sustancias no húmicas (CSNH), fue alrededor del $70 \%$; el carbono orgánico total correlacionó mejor con carbono orgánico extraíble con 86\%; el carbono orgánico extraíble presentó correlación con las sustancias no húmicas en $72 \%$. Entre los índices, no hubo correlaciones claras entre ellos, lo cual se debe a que en estos suelos intervienen muchos factores en la dinámica de la descomposición de la MOS, entre ellos la pendiente, tipo, cantidad y frecuencia de materia orgánica que se aplica, etc.

\section{CONCLUSIONES}

El uso intensivo de la tierra en labores agrícolas disminuyen los contenidos de carbono orgánico en las fracciones químicas como los ácidos húmicos, ácidos fúlvicos y sustancias no húmicas y por tanto altera también los índices de humificación.

Se encontró un alto porcentaje de materia orgánica humificada en todos los usos, sin embargo, hay una tendencia de aumento en los compuestos de menor grado de polimerización al intervenir el suelo.

Gran parte de las pérdidas de la materia orgánica del suelo (MOS) fueron causadas por la erosión ayudada por las altas pendientes del terreno, condición a tener presente en los planes de reposiciones de MOS. 


\section{REFERENCIAS}

Acosta, Y., Paolini, J. y Benítez, E. 2004. Índice de humificación y prueba de fitotoxicidad en residuos orgánicos de uso agrícola potencial. Revista Facultad de Agronomía (LUZ) 21(4):185-194.

Anderson, J. M. e Ingram, J. S. I. 1993. Tropical Soil Biology and Fertility. A Handbook of Methods. $2^{\text {nd }}$ ed. Wallingford, $C A B$ International, UK, p221.

Armado, A.; Contreras, F. y García, P. 2009. Fraccionamiento químico de carbono orgánico y su relación con la actividad microbiológica, biomasa microbiana y cantidad de ADN en suelos cacaoteros venezolanos. Revista Sociedad química del Perú, 75(1):44-53.

Avilán, L., Brendler, A. y Hernaiz, A. 1975. Evaluación de los suelos y del estado nutricional del cultivo del durazno (Prunus persica L.) en la Colonia Tovar. Agronomía Tropical 25(1): 91.

Figuera, K. C.; Lozano, Z. y Rivero, C. 2005. Caracterización de diferentes fracciones de la materia orgánica de tres suelos agrícolas venezolanos. Venesuelos 13:34-46.

Galantini, J. y Suñer, L. 2008. Soil organic matter fractions. Analysis of Argentine soils. Agriscientia, XXV(1): 41-55.

Heanes, D. 1984. Determination of total organic- $\mathrm{C}$ in soil by an improved chromic acid digestion and spectrophotometric procedure. Revista Soil Science and Plant Analysis. 15:1191-1213.

Herrera, F. y Cuevas, E. 2003. Artrópodos del suelo como bioindicadores de recuperación de sistemas perturbados. Venesuelos 11:67-78.

Jaimes, E. 1988. Determinación de índices homogeneidad múltiples globales en sistema pedogeomorfológicos de la Cordillera de la Costa Serranía del Litoral Central. Tesis Doctor en Ciencias del Suelo, Facultad Agronomía, programa de postgrados en Ciencias del Suelo, Universidad Central de Venezuela, Venezuela.

López, R.; Gallegos, A.; Peña, E.; Reyes, A.; Castro, R. y Chávez, J. 2006. Substancias húmicas de origen diverso en algunas propiedades físicas de un suelo francoarcillo-limoso. Revista Terra, 24(3): 303309.

Lozano, Z.; Bravo, C.; Hernández, R.; Del'abate, M.; Alianelo, F. y Benedetti, A. 2002. Efecto de cultivos de cobertura de diferentes calidades sobre la materia orgánica de dos suelos Venezolanos. Venesuelos $10(1$ y 2): 47-60.

Machado, C., Vargas, R., Pinheiro, D., Pauletto, E. y Da Silva, A. 2008. Teor e qualidade de substâncias húmicas de planossolo sob diferentes sistemas de cultivo. Revista Ciencia Rural, 38(6): 1589-1595. 
Mogollón, L. Y Comerma, J. 1994. Suelos de Venezuela. Gerencia Corporativa de Asuntos Públicos de PDVSA Palmaven, Caracas, Venezuela. p313.

Piccolo, G., Rosell, R., Galantini, J. y Miglierina, A. 1998. Transformaciones de la materia orgánica en un suelo laterítico (Misiones, Argentina), II cambios en la materia orgánica particulada y humificada. Revista Agricultura técnica (Chile), 58(2): 142-150.

Pulido-Moncada, M.; Flores, B.; Rondón, T.; Hernández-Hernández, R. y Lozano, Z. 2010. Cambios en fracciones dinámicas de la materia orgánica de dos suelos, Inceptisol y Ultisol, por el uso con cultivos de Cítricas. Bioagro, 22(3): 201 210.

Romanyà, J., Rovira, P. y Vallejo, R. 2007. Análisis del carbono en los suelos agrícolas de España. Aspectos relevantes en relación a la reconversión a la agricultura ecológica en el ámbito mediterráneo. Revista Ecosistemas, 16(1): 50-57.

Ruiz, M., ElizaldE, G. y Paolini, J. 2000. Correlaciones entre el carbono orgánico de los microagregados y algunos atributos del suelo en paisaje de sabana. Revista Facultad Agronomía (Maracay), 26:125-135.
Sánchez, J. 1981. Mesoclimas en Venezuela. Ministerio de Agricultura y Cría. (mapas). FONAIAP. CENIAP. Caracas. p33.

Sánchez, B., Ruiz, M. y Ríos, M. 2005. materia orgánica y actividad biológica del suelo en relación con la altitud, en la cuenca del río Maracay, Estado Aragua. Agronomía Tropical, 55(4): 507-534.

Schinitzer, M. y Schuppli, P. 1989. Methods for sequential extraction of organic matter from soils and soil fractions. Revista Soil Science Society of America Journal, 53:1418-1424.

Zagal, E. y Córdova, C. 2005. Indicadores de Calidad de la Materia Orgánica del Suelo en un Andisol Cultivado. Agricultura Técnica (Chile), 65(1):186-197.

Zalba, P. y Quiroga, A. 1999. Fulvic Acid Carbon As A Diagnostic Feature for Agricultural Soil Evaluation. Soil Science 164(1):57-61. 